\title{
Decision Analysis Concerned With Investments in Fire Safety
}

\author{
Henrik Johansson \\ Department of Fire Safety Engineering, Lund University \\ P.O. Box 118, SE-221 00 Lund, Sweden
}

\begin{abstract}
Decision analysis concerned with investments in fire safety is discussed. Particular attention is directed at the treatment of uncertainty, the evaluation of consequences, and the choice of a decision rule for use in this context. An approach involving use of a decision rule based on the principle of maximising expected utility, together with a complementary evaluation of the decision alternatives, is described, the latter involving analysis of the uncertainty regarding the probabilities and consequences of different fire scenarios.
\end{abstract}

KEY WORDS: Decision analysis, Bayesian methods, uncertainty, fire safety.

\section{INTRODUCTION}

The management of an organisation has the obligation towards the shareholders and other interested parties, of managing effectively any risks that can threaten the organisation's goals. This involves making decisions concerning risk-reducing investments such as investments in fire safety. The present paper deals with various aspects of decision analysis concerned with investments in fire safety, both a decision rule and a method for performing such an analysis being suggested. The paper will focus on the choice between different fire protection alternatives for a given building. Note that what is of interest here is the choice between decision alternatives, not the attempt to determine whether a given decision alternative possesses certain necessary or desired properties, as would be the case if one employed decision analysis to investigate whether an alternative met the requirements of the building codes that apply. It is assumed that all fire protection alternatives that are considered comply with the building codes that are applicable.

In the following section, the connection between risk analysis and decision analysis is discussed. The practical benefits are pointed out of using a risk analysis of a particular building as a point of departure when performing a decision analysis regarding possible fire protection measures for a building. Section 3 deals with the treatment of uncertainty. Section 4 is concerned with estimating and evaluating consequences within a decision analysis. Section 5 deals with decision rules for the analysis of different fire protection alternatives. In section 6 , the decision method suggested is presented, together with a real-world example. In section 7, finally, a number of conclusions are drawn regarding the use of decision analysis for evaluating fire safety investment alternatives.

\section{RISK ANALYSIS}

It is assumed that the decision analysis is based on a quantitative risk analysis. If the general framework for fire-risk analysis outlined by Hall and Sekizawa [1] is employed, for example, the "fire risk" involved or the "outcome measure of fire risk", is defined according to Eq. 1. The term $g\left(s^{\prime}\right)$ in Eq. 1 is a function that transforms the severity 
measure $s^{\prime}$ into the measure of interest in the risk analysis. For example, if $s^{\prime}$ is the monetary loss due to a specific fire scenario and the measure of interest is monetary losses, then $g\left(s^{\prime}\right)=s^{\prime} . P\left(s=s^{\prime}\right)$ in Eq. 1 refers to the probability that the severity measure $s^{\prime}$ will occur.

$$
\text { Risk }=\int_{-\infty}^{+\infty} g\left(s^{\prime}\right) P\left(s=s^{\prime}\right) d s^{\prime}
$$

Since in practice it is likely that the risk measure will be based upon a finite set of fire scenarios, Eq. 1 can be replaced by Eq. 2, in which $n$ is the number of fire scenarios that is deemed to be relevant in the building in question.

$$
\text { Risk }=\sum_{i=1}^{n} g\left(s_{i}\right) P\left(s=s_{i}\right)
$$

If one performs a risk analysis using Eq. 2 one must have a number of different fire scenarios that have been defined, together with the outcome measure and the probability of occurrence for each scenario.

In using risk analysis as a point of departure for the decision analysis to be carried out, there are (at least) three areas in which difficulties are likely to be encountered. These are the evaluation of consequences for each of the different fire scenarios, treatment of the uncertainty in the probability estimates and in the estimates of consequences, and the choice of a decision rule. The choice of a decision rule is dependent upon the methods one elects to use in performing the other tasks, and it is also that aspect of decision analysis with which the present paper is most concerned. Because of its dependence on the other aspects of decision analysis, it will be taken up last.

\section{MANAGING UNCERTAINTY}

The first difficulty in connection with decision analysis to be discussed here is that of dealing with uncertainty regarding both the consequences and the probabilities associated with them. When probabilities are used to describe uncertainty, it is necessary to first define how the probability concept is to be conceived. The interpretation of probability with respect to risk analysis has been discussed in [2], and with respect to fire-risk analysis in [3]. Both authors involved suggest use of a subjective interpretation of probability, meaning that probability is regarded as a measure of degree of belief. In the present paper, the subjective interpretation of probability will be adopted. The reason for this is (1) that this interpretation is used in the Bayesian decision theory, which is the theory employed in the present paper and (2) it gives a flexibility to use other kinds of information than purely empirical information, such as expert judgement, for example.

In endeavouring to estimate the probabilities of each of the uncertain events that affect the outcome of a fire, it is often difficult to assign precise values to the probabilities in question. This is because one usually does not have sufficient information regarding any given probability to feel comfortable in expressing one's degree of belief as a single value. Instead, using a set of plausible values or an interval may seem more adequate. From a Bayesian point of view, uncertainty regarding a specific probability value is 
expressed as a probability distribution representing one's degree of belief regarding the different probability values (See [4], for example). In expressing one's belief regarding a particular probability as a probability distribution, one can use Bayes' theorem to incorporate new information into one's initial belief.

Bayesian methods can also be used to help the decision maker incorporate information from other sources than those of his/her own judgements into the analysis, such as expert judgements or fire statistics (see, [5], for example). In many cases, this helps considerably in reducing the uncertainty (making the distribution less broad) regarding the value of the probability in question. Although a large reduction in uncertainty can be achieved by use of Bayesian methods, one still ends up with a distribution of probability values that one needs to somehow make use of in the decision analysis. How such probability distributions are dealt with in decision analysis is discussed in the section concerned with decision rules.

\section{EVALUATION OF CONSEQUENCES}

The consequences of a fire can be expressed in many different ways, such as the number of people whose health was affected by it, the value of the physical property that the fire destroyed, or whatever. In analysing different fire protection alternatives in a building that belongs to some particular organisation it is often convenient, assuming that all alternatives comply with the building codes, to endeavour to assess the damage due to a fire in terms of the intrinsic (negative) monetary value of the consequences as viewed by the decision maker. Methods of differing degrees of sophistication can be used to arrive at this intrinsic monetary value. One could use multi-attribute utility theory (see e.g. [6]) for example, to arrive at the intrinsic monetary value of each possible set of consequences, or one could settle for less formal models and simply try to evaluate the intrinsic monetary value for each fire scenario directly, without use of any formal approach to the problem. One reason for using intrinsic monetary values to obtain measures of relative preference for the different possible sets of consequences is that the monetary scale is one that people are accustomed to, its thus providing an effective means of communicating how good or bad the decision maker judges a particular outcome of a fire to be.

In practice, one needs to decide which losses that should be part of the evaluation. Obviously, monetary losses the decision maker is reimbursed for in case of fire should not be treated as losses in the decision analysis. However, one needs to be careful in considering the effects of insurance. Even with good insurance coverage there may be losses the decision maker will not be reimbursed for. In [7] it is indicated that only some $40-60 \%$ of the actual losses due to a disaster are covered by insurance. Although the amount of the losses covered by insurance obviously depends upon the building involved and the insurance covering it, it is important to remember that considerable losses for the owner may occur, even if the building has good insurance coverage.

A term that can be used to denote all losses due to a fire, including losses due to business interruption, that the owner eventually has to defray is that of uncompensated losses. Such losses can include lost market shares, fines, negative reputation, and the like. It is very difficult to provide any general guidelines for the types of losses to be included in the calculations. Rather, that needs to be investigated in the specific case. Once the uncompensated losses have been identified, one needs to estimate their intrinsic monetary 
value. In doing so it is very likely, just as it was for the probabilities discussed above, that one will feel uncertain regarding the value to use. Instead of expressing the value as a precise number, it may be better to use an interval or a probability distribution to represent one's belief regarding the plausibility of the different values.

In working with practical applications, it is not always feasible to perform a complete analysis of uncompensated losses, since this could involve disproportionate work efforts in relation to the importance of the decision. It is useful, therefore, to distinguish between different levels of analysis, the level chosen depending on how thoroughly the uncompensated losses are to be investigated. A suggestion for how these levels of analysis can be defined is provided in [8]. As indicated there, an analysis of fire safety investment can be performed on at least three levels, that of (1) ignoring the increase in safety and of basing an evaluation of the investment on parameters one is basically certain about, such as investment costs, reduction in insurance premiums, maintenance costs, etc., (2) taking account of all costs (and benefits) at level 1 and adding to this the valuation of the risk reduction achieved by using a subset of the uncompensated losses in the consequence estimations or any other losses for which the relation they have to the uncompensated losses can be assessed, or (3) taking all losses at level 1 into account and attempting to estimate all the uncompensated losses of importance.

Although which of the levels required depends on the problem at hand, it could be wise to start an analysis at level 1 and then increase the level of analysis if it is deemed necessary, since a higher level of analysis generally requires more work. A higher level of analysis tends to "favour" decision alternatives representing safety investments, since such investments generally decrease the probability of some of the fire scenarios that have serious consequences and generally includes large uncompensated losses.

\section{DECISION RULES}

Having discussed some of the major problems and some of the possible ways of estimating the probabilities and consequences involved in a decision analysis, one needs to also consider the basis for evaluating the different decision alternatives.

In order to find a suitable decision rule, Bayesian decision theory will be examined to see whether that theory can prove useful in the present fire engineering context. The applicable decision rule for Bayesian decision theory is the principle of maximising expected utility. This is a principle that has been used extensively in the context of engineering (see [9], for example) and it has also been used in fire engineering (see [10], for example).

Modern decision theory has its roots in work performed by Ramsey [11], von Neumann and Morgenstern [12] and Savage [13], in particular. In these references, axiomatic systems for comparing preferences for different acts with uncertain outcomes have been formulated. The basic approach taken in constructing such axiomatic systems is to formulate a number of rules (axioms) that seem intuitively reasonable for comparing preferences between different acts with outcomes that are uncertain. From these axioms, a number of important results can then be derived, such as the principle of maximising expected utility (MEU). The MEU principle implies that a person who is willing to follow these axioms in his/her decision making will evaluate decision alternatives according to their expected utility and choose the decision alternative with the highest 
expected utility. Of the authors referred above, Savage has been called the principal founder of modern decision theory [14], which is also termed Bayesian decision theory. A review of the various theories of this type and of major aspects of modern decision theory have been provided by Fishburn [14].

Before discussing whether the MEU principle is reasonable to employ in the present context, it is useful to review some of the criticism that have been directed against axiomatic systems of the type that Savage proposes. The criticism that are discussed here are of two types: criticisms based on empirical investigations and criticisms directed against the logical foundations of the MEU principle.

In the first category, criticisms based on empirical investigations, the perhaps most famous criticisms of Bayesian decision theory are those made by Allais [15] and by Ellsberg [16]. Of these two authors, the one whose criticism is most relevant in the present context of fire safety would seem to be Ellsberg. Ellsberg's basic criticism is that in making choices between decision alternatives with uncertain outcomes, uncertainty regarding the probabilities and the value of the outcomes appears to influence how people choose. This type of uncertainty should, according to the Bayesian decision theory, not matter for a decision. According to this theory the uncertainty regarding probability values and consequence values should be expressed in terms of probability distributions representing the decision maker's belief regarding these values. In evaluating decision alternatives within a Bayesian framework uncertainty regarding the probabilities (how spread the distribution representing one's belief is) does not affect the decision, the only thing used in the evaluation of decision alternatives being the expected value (mean) of the distribution representing one's degree of belief. In the present context, nevertheless, it is desirable to be able to distinguish between situations in which a decision maker is very certain regarding his/her probability estimates and one in which he/she is not. For this reason, the term "robust decision" is introduced. Robust decisions will be discussed shortly.

The second category of criticism concerns the logical foundation of the MEU principle. Malmnäs [17] shows that the axiomatic systems proposed by Savage [13], among others, is too weak to imply the MEU principle. This is a serious criticism since it suggests that there are other decision criteria besides the MEU principle that satisfy the axioms and that the MEU principle is thus not a logical consequence of having accepted the axioms. Malmnäs undermines in this way one of the strongest arguments for using the MEU principle as a decision rule, namely that by accepting the axioms as rules for one's decision making one will then act as if one were evaluating decision alternatives according to their expected utility. In another paper, Malmnäs [18] examines the extent to which it is possible to provide the MEU principle support in a different way, that of showing that the rule does not give rise to counter-intuitive choices to any appreciable extent, counter-intuitive in the sense of a decision rule's evaluating an decision alternative with uncertain outcomes in a way not supported by human intuition. Although Allais [15], for example, has provided examples of situations in which the MEU principle generates counter-intuitive choices, Malmnäs concludes that any simpler rule than the MEU principle gives rise to counter-intuitive choices to a greater extent than the MEU principle does and that "...the prospects for finding an evaluation [decision rule] that is much better than $\mathrm{E}(\mathrm{A}, \mathrm{f})[\mathrm{MEU}]$ are not particularly bright.”. 
As was indicated above, although considerable criticism has been directed against the MEU principle, this decision rule still appears to be a strong candidate for being a decision rule that can be used in connection with a quantitative risk analysis and at the same time is practical for use in the present context. The rule may possibly be in need of slight modification or require a complimentary evaluation of decision alternatives so that those alternatives involving uncertainty regarding the probabilities and consequences can be recognised. One way of doing this is to first evaluate all the decision alternatives using the MEU criterion, so as to find the decision alternative with the highest expected utility, which can be termed "the MEU alternative". When this decision alternative has been identified, it should be compared with the other decision alternatives in terms of the uncertainty connected with the estimates of probabilities and of consequences. One way of doing this would be to relate the uncertainty regarding the probabilities and consequences to the value of the expected utility. Relating the uncertainty regarding the probabilities and consequences to the value of the expected utility involves the expected utility no longer being expressed as a single value but as a probability distribution. Thus, comparing the MEU alternative with the other decision alternatives involves comparing probability distributions rather than precise values.

In comparing the alternatives in terms of the uncertainty connected with the estimates of probabilities and of consequences one is interested in the difference in expected utility. Since the expected utility of a decision alternative is expressed as a probability distribution, the difference in expected utility between two decision alternatives is also a probability distribution. Expressing the difference in expected utility in this way makes it possible to visualise the uncertainty regarding the value the difference has, and to take account of this in the decision to be made. If the major part of the mass of the probability distributions illustrating the difference in expected utility between the MEU alternative and the other decision alternatives indicates the MEU alternative to be best, then the decision is said to be robust, its otherwise being deemed not robust. What the "major part" in the above sentence means is up to the individual decision maker to decide. $\mathrm{He} /$ she might assume, for example, that a decision is robust if $95 \%$ of the resulting distribution representing the difference in utility between two decision alternatives indicates the MEU alternative to be best. The concept of a robust decision is introduced here to provide an indication of how likely it is that the recommended decision alternative (the MEU alternative) will change if a plausible degree of change in the probabilities and the consequences should be made. To exemplify such an approach, consider a choice between three fire protection alternatives for which the uncertainty regarding the values of the probabilities and of the consequences in the model is expressed as distributions that represent the decision maker's belief regarding their values. Assume in addition that the result when calculating the expected utility of the different decision alternatives is that alternative 1 has the highest expected utility, followed by alternative 2 and alternative 3 in that order. Thus, according to Bayesian decision theory, alternative 1 is the decision alternative the decision maker should choose. Assume, however, that there is not much that differs between alternative 1 and alternative 2, and that in comparing the two decision alternatives in terms of the difference in the expected utility $\left(E\left(U_{1}\right)-E\left(U_{2}\right)\right)$ and expressing the difference as a probability distribution, one can see that a slight change in the decision maker's belief could lead to alternative 2 being the best decision alternative, as shown in the distribution termed $\mathrm{A}$ in Fig. 1. In that figure, the area of the probability distribution to the left of the 0 value on the horizontal axis implies that alternative 2 is best, since $E\left(U_{1}\right)-E\left(U_{2}\right)$ is negative there. In this case, the decision to choose alternative 1 
would probably not be considered to be robust since a large part of the probability distribution termed A in Fig. 1 implies that alternative 2 is best. In contrast, if one looks at the distribution representing the difference in expected utility between alternative 1 and alternative 3, as shown in the distribution termed B in Fig. 1, one notes that the situation is quite different. There, the whole probability distribution representing the difference in expected utility between the decision alternatives $\left(E\left(U_{1}\right)-E\left(U_{3}\right)\right)$ is within the positive region on the horizontal scale, so that if alternative 1 and 3 are the only decision alternatives to choose between, deciding for alternative 1 would be considered a robust decision.
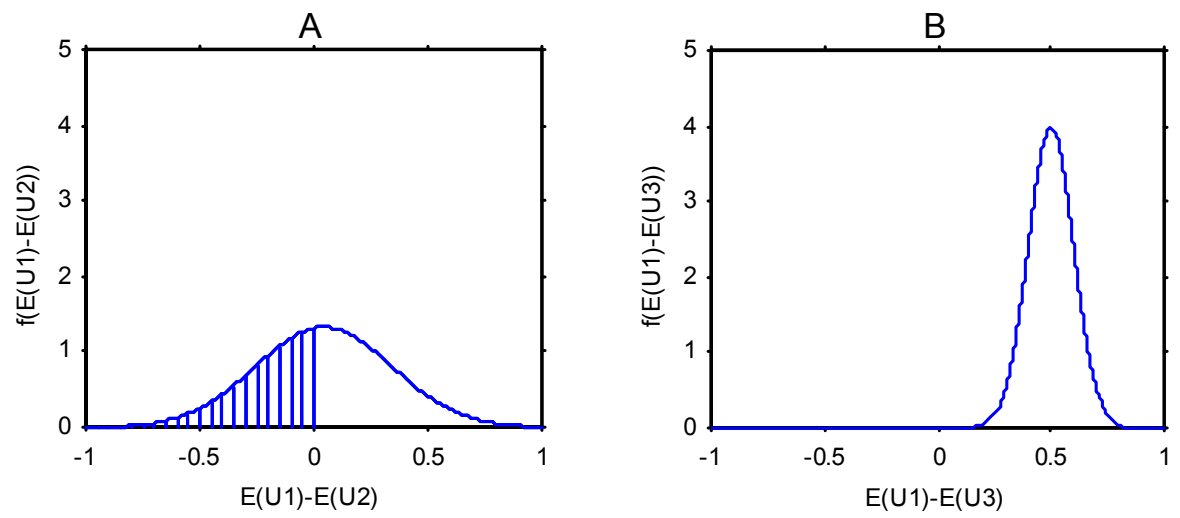

Fig. 1

Probability distribution representing the difference in expected utility between alternative 1, 2 and 3.

\section{SUMMARY OF THE APPROACH}

The approach suggested here for decision analysis concerned with investments in fire safety is based on the extension of Bayesian decision theory presented in the previous section. The treatment of uncertainty and the quantification of consequences were discussed in section 3 and 4.

A real-world decision analysis will be used to exemplify the approach taken. The analysis in question was conducted in 1998 at a firm called Asea Brown Boveri (ABB). It concerned the possible investment in a sprinkler system for a building belonging to the company. At the time, ABB was producing circuit cards for use in their robots and automation systems in the building. The building was approximately $55.000 \mathrm{~m}^{2}$ in size. Since the analysis was quite an extensive one, involving more than 150 different fire scenarios, only selected parts of it will be discussed. See Ref. 8 for a more comprehensive account.

The first step in conducting the type of decision analysis described here (see Fig. 2, step 1) is to identify the decision alternatives involved and decide upon the time period of concern. In the ABB case, there were only two decision alternatives: (1) keeping the building in its current state and (2) investing in a water sprinkler system for the building as a whole. The time period decided upon was one of 40 years. To determine whether choice of this particular time period had any effect on which alternative was deemed best, the same analysis was conducted for periods of 5 years, 10, etc., its being concluded that the length of the time period had no effect. 
The next step is to determine what level of analysis to employ (step 2 in Fig. 2). This involves deciding which losses are to be treated as uncompensated ones. In the ABB case, it was decided that the total costs of the equipment destroyed and of the interruption in business that a particular fire scenario entailed would be considered as uncompensated losses. Although ABB would later be reimbursed for the loss of equipment and for a part of the costs of the business interruption this sum was judged to be an appropriate measure of the total uncompensated losses as seen in monetary terms. The analysis as a whole was performed in accordance with the level 2 definition given in Section 4.

1. Define the alternatives and specify the time period of interest. 2. Choose the level of analysis (i.e. decide which losses to regard as
uncompensated losses).

3. Determine the costs of each of the alternatives.

For each alternative:

-Estimate the probability of the different fire scenarios.

4. - -Determine the uncompensated losses for each fire scenario.

-Calculate the expected utility (or Certainty Equivalent) for the time period of interest.

5. Determine which alternative is the MEU alternative (i.e. the one with
the highest expected utility).

Determine how the uncertainty of the probabilities and of the

6. consequences is related to the difference between the alternatives in terms of expected utilities (or Certainty equivalent).

7. Determine robustness.

Fig. 2

The method of decision analysis suggested for decisions concerning investments in fire safety.

Since the sprinkler system was the only investment considered in the ABB case, only costs associated with that system needed to be included in the analysis. The sprinkler system was estimated to cost $\$ 1.000 .000$ and annual maintenance of it $\$ 10.000$ (step 3 in Fig. 2).

The next step is to perform a risk analysis of each of the decision alternatives with the aim of identifying a set of fire scenarios, their respective probabilities of occurrence and their consequences in terms of uncompensated losses. This could be achieved, for example, by use of an event tree technique in which the uncertain events judged to affect the outcome of the fire are modelled. Which events to include in the event tree depends 
very much on the building at hand and the level of detail aimed at. In the ABB case, for example, events involving the sprinkler system and the fire detection system, as well as the building occupants and the fire department were used in the event trees. Evaluating the uncompensated losses involves estimating a monetary value that is seen as equal to each of the consequences. In the $\mathrm{ABB}$ case, this was accomplished by having the analyst explain a particular fire scenario, in terms of the extent of fire spread, to people from $\mathrm{ABB}$ and having them estimate the effect of such a fire in terms of uncompensated losses. Examples of uncompensated losses associated with some of the fire scenarios considered in the ABB case are given in Table 1. One can see that, so as to express the uncertainty involved, numbers are given there representing the most likely, the minimum and the maximum value respectively, for the consequences in question. These values are used to create triangular probability distributions to represent the decision maker's beliefs regarding the losses to be expected.

Table $1 \quad$ The uncompensated losses associated with different fire scenarios in the $A B B$ case. The fire scenarios apply to a fire compartment in which an electronic workshop is located.

\begin{tabular}{|l|c|c|c|}
\cline { 2 - 4 } \multicolumn{1}{c|}{} & \multicolumn{3}{c|}{$\begin{array}{l}\text { Uncompensated losses associated with the } \\
\text { fire scenario in question (\$ thousand) }\end{array}$} \\
\hline Fire scenario & Minimum & $\begin{array}{c}\text { Most } \\
\text { likely }\end{array}$ & Maximum \\
\hline $\begin{array}{l}\text { A fire is extinguished either by } \\
\text { employees or by the sprinkler system. }\end{array}$ & 5 & 10 & 15 \\
\hline $\begin{array}{l}\text { A fire of limited scope is extinguished } \\
\text { by the fire department. }\end{array}$ & 25 & 50 & 75 \\
\hline $\begin{array}{l}\text { An extensive fire is extinguished by the } \\
\text { fire department. }\end{array}$ & 533 & 1067 & 1933 \\
\hline $\begin{array}{l}\text { A fire completely destroys the fire } \\
\text { compartment. }\end{array}$ & 25920 & 32000 & 38720 \\
\hline
\end{tabular}

The next step is to evaluate the different decision alternatives. The basis for doing so was discussed in the previous section, where it was concluded that the maximisation of expected utility is the decision rule applicable here. The expected utility of a decision alternative can be used to calculate the Certainty equivalent (CE) of it. The CE is a monetary sum equal in value to that of some particular situation involving uncertainty (see [19]). In this case, the $\mathrm{CE}$ is the (negative) monetary amount equal in value to choosing a particular fire protection alternative, including the costs of the alternative and the possibility of having one or more fires in the building during the time period of interest (see Fig. 3). The CE can be considered to be a better unit than "expected utility" for comparing decision alternatives, since it is expressed in terms of monetary value and people are more likely to feel comfortable using monetary sums than using expected utilities for comparison purposes. Note that whether expected utility or CE is used for comparing the decision alternatives should not affect the end result, the alternative being recommended being the same in both cases.

In order to calculate a $\mathrm{CE}$ here, one needs first to estimate how frequently fires will occur. Estimating the frequency of fires in the ABB building involved use of Bayesian methods, utilising the information that four fires altogether had occurred there during the 
years of 1996, 1997 and 1998. Using the estimate of fire frequency arrived at, together with the event tree presenting the different fire scenarios, the costs of the sprinkler alternative, and the uncompensated losses associated with each fire scenario made it possible to estimate the CE for each of the two decision alternatives (step 4. in Fig. 2). This involves calculating the expected utility of one fire and then multiplying this value with the expected number of fires during the time period of interest. Note that one can regard losses occurring late in the period of interest as being less severe than those occurring earlier. This is discussed in detail in [20].

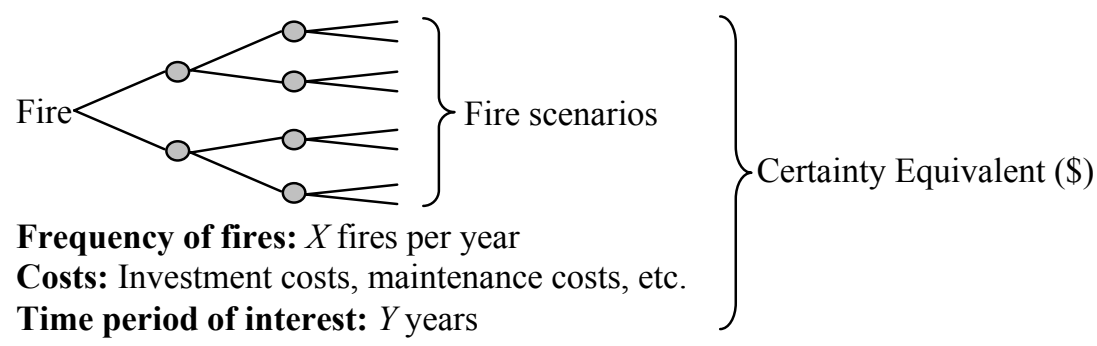

Fig. 3

Illustration of the calculation of the Certainty Equivalent of a decision alternative.

The results of the CE calculations indicated the decision alternative of investing in a sprinkler system to have the highest CE, and thus the highest expected utility (step 5. in Fig. 2).

In order to determine whether the decision of choosing the sprinkler alternative was robust, the question of how the knowledge uncertainty concerning the probabilities and the consequences was related to the difference in CE between the MEU alternative (the sprinkler alternative in the $\mathrm{ABB}$ example) and the other decision alternative was investigated. Since many of the probabilities and consequences used to calculate the $\mathrm{CE}$ (Expected utility) are uncertain, the $\mathrm{CE}$ is also uncertain. In the analysis, there were over 100 probabilities and consequences with a significant uncertainty regarding their values. One of those was the probability that the occupants would extinguish a fire in a particular storage room given that the smoke detection system functioned as intended. This conditional probability was estimated to be somewhere between 0,2 and 0,6 , with a most likely value of 0,4 . If the smoke detection system did not function as intended the probability was estimated to be somewhere between 0,1 and 0,3 with a most likely value of 0,2 . This kind of uncertainties was modelled using triangular distributions representing the probabilities when the calculation of the $\mathrm{CE}$ was performed. By use of Monte Carlo simulation (5000 iterations), the histogram presented in Fig. 4, showing the differences between the two decision alternatives in terms of CE could be obtained (step 6. in Fig. 2).

Figure 4 illustrates that the decision to invest in a sprinkler system for the ABB building is robust (compare Fig. 4 to Fig. 1) since all the values from the Monte Carlo simulation indicate that the sprinkler alternative has the highest CE (step 7. in Fig. 2). Figure 4 also shows that the difference between the two decision alternatives, in terms of $\mathrm{CE}$, is substantial. The mean value of the difference is approximately $\$ 3,1$ million. Two reasons for the large difference in $\mathrm{CE}$ is that a serious fire in the building would cause significant 
losses for ABB (if the whole building is destroyed the uncompensated losses would be in the order of several hundred million dollars) and that the standard of the fire protection in the buildings original design were poor.

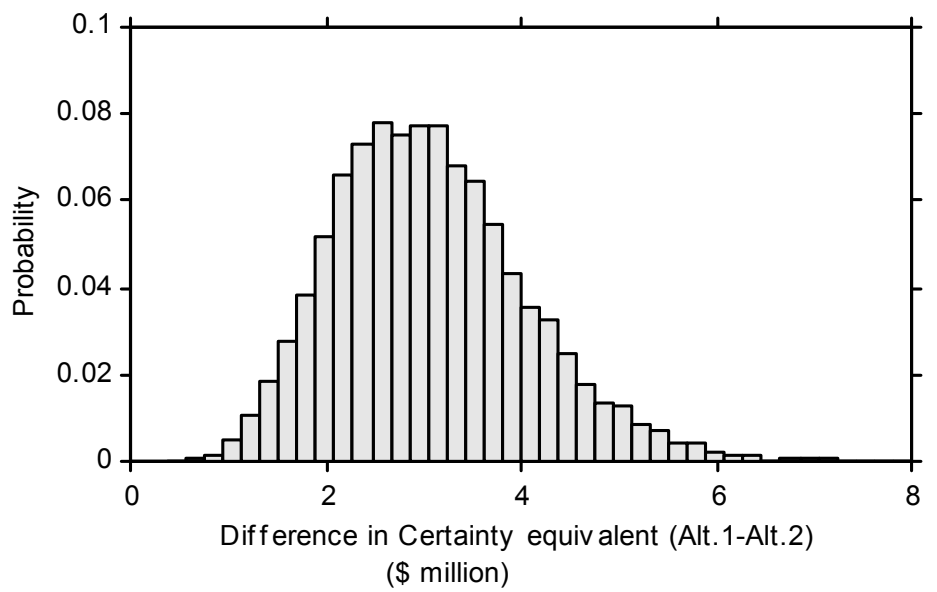

Fig. 4

The differences in $C E$ in the $A B B$ example. The CE of alternative 2 (not to invest in a sprinkler system) is subtracted from the CE of alternative 1 (to invest in a sprinkler system).

\section{CONCLUSIONS}

Decision analysis as applied to problems in which a decision maker is to decide between different fire protection alternatives for a particular building have been discussed.

In connection with estimating the possible consequences of a particular fire scenario, the concept of uncompensated losses was defined as the losses that the decision maker or organisation in question eventually have to defray. Since it is often practical to express such losses as monetary consequences, the intrinsic monetary value of the uncompensated losses generally needs to be estimated and to be used in the analysis.

The question of what decision rule should be used in the present context was discussed. Use of the criterion of maximising expected utility (MEU) being recommended. Various of the, major criticisms of use of this criterion in the present context were presented. In view of this criticism, it was considered to be advantageous to complement the MEU criterion with an evaluation of the robustness of the decision. A robust decision alternative was defined as an alternative that in terms of the MEU criterion remained the preferred one for most of the combinations of plausible probability and utility values that could be identified.

A real-world problem involving the evaluation of an investment in a water sprinkler system was presented. The building in which the investment was considered belonged to the company ABB. The investment in a water sprinkler system was found to be the best alternative. It was also concluded that the decision to invest in a sprinkler system was robust. 


\section{ACKNOWLEDGEMENTS}

The author would like to thank The Swedish Fire Research Board (BRANDFORSK) for funding the research on which the present paper is based.

\section{REFERENCES}

1. Hall, J.R., Sekizawa, A., "Fire Risk Analysis: General Conceptual Framework For Describing Models", Fire Technology 27: 33-53 (1991).

2. Apostolakis, G., "Probability and Risk Assessment: The Subjectivistic Viewpoint and Some Suggestions", Nuclear Safety 19: 305-315 (1978).

3. Noonan, F., Fitzgerald, R., "On the Role of Subjective Probabilities in Fire Risk Management Studies”, Fire Safety Science - Proceedings of the Third International Symposium, International Association for Fire Safety Science, 1991, pp. 495-504.

4. Lindley, D.V., Introduction to Probability and Statistics From a Bayesian Viewpoint, Part 2: Inference, Cambridge University Press, Cambridge, 1965.

5. Sui, N., Apostolakis, G., "Uncertain Data and Expert Opinions in the Assessment of the Unavailability of Suppression Systems", Fire Technology 24: 138-163 (1988).

6. Keeney, R.L., Raiffa, H., Decisions with Multiple Objectives: Preferences and Value Tradeoffs, Wiley, New York, 1976.

7. Hiles, A., "Marketing Protection: a justification for funding of Total Asset Protection programmes?", The Definite Handbook of Business Continuity Management, Hiles A., Barnes P. (eds.), John Wiley \& Sons, Chichester, 1999, pp. 67-73.

8. Johansson, H., Decision Making in Fire Risk Management, Report 1022, Department of Fire Safety Engineering, Lund University, Lund 2001.

9. Benjamin, J.R., Cornell, C.A., Probability, Statistics, and Decision for Civil Engineers, McGraw-Hill Book Company, New York, 1970.

10. Shpilberg, D., De Neufville, R., "Best Choice of Fire Protection: An Airport Study", Fire Technology 10: 5-14 (1974).

11. Ramsey, F.P., "Truth and Probability", The Foundations of Mathematics and Other Logical Essays, Routledge and Kegan Paul, pp. 156-198, London, 1931. Reprinted in H. E. Kyburg and H. E. Smokler (Eds.), Studies in subjective probability, Wiley, New York, 1964, pp. 61-92.

12. Von Neumann, J., Morgenstern, O., Theory of Games and Economic Behaviour, Princeton University Press, NJ, 1944.

13. Savage, L.J., The Foundations of Statistics, Wiley, New York, 1954.

14. Fishburn, P.C., "Foundations of Decision Analysis: Along the Way", Management Science 35: 387-405 (1989).

15. Allais, M., "Le comportement de I'homme rationnel devant le risque: Critique des postulats et axioms de lécole americaine", Econometrica 21: 503-546 (1953).

16. Ellsberg, D., "Risk, ambiguity, and the Savage axioms", Quarterly Journal of Economics 75: 643-669 (1961).

17. Malmnäs, P.-E., "Axiomatic Justifications of the Utility Principle: a Formal Investigation", Synthese 99: 233-249 (1994).

18. Malmnäs, P.-E., Methods of Evaluation in Supersoft Decision Theory, Department of Philosophy, Stockholm University, 1995.

19. Clemen, R.T., Making Hard Decisions - An Introduction to Decision Analysis, $2^{\text {nd }}$ ed., Duxbury Press, Belmont, 1996, p. 469.

20. Johansson, H., "Investment Appraisal Using Quantitative Risk Analysis", Journal of Hazardous Material, Accepted for publication. 